\title{
Systematic study of quasifission characteristics and timescales in heavy element formation reactions
}

\author{
D.J. Hinde ${ }^{1}$, E. Williams, G. Mohanto ${ }^{2}$, C. Simenel, M. \\ Dasgupta, A. Wakhle ${ }^{3}$, I.P. Carter, K.J. Cook, D.Y. Jeung, \\ D.H. Luong, C.S. PalshetkaR ${ }^{4}$, E. Prasad, D.C. Rafferty, \\ R. DU Rietz ${ }^{5}$ and E.C. Simpson
}

Department of Nuclear Physics, Research School of Physics and Engineering, Australian National University, ACT 2601, Australia

\begin{abstract}
Superheavy elements can only be created in the laboratory by the fusion of two massive nuclei. Mass-angle distributions give the most direct information on the characteristics and time scales of quasifission, the major competitor to fusion in these reactions. The systematics of 42 mass-angle distributions provide information on the global characteristics of quasifission. Deviations from the systematics reveal the major role played by the nuclear structure of the two colliding nuclei in determining the reaction outcome, and in hindering or favouring heavy element production.
\end{abstract}

To form very heavy and superheavy elements (SHE), heavy-ion fusion reactions are used. Their cross sections can be significantly suppressed [1] by quasifission [2]. This dynamical non-equilibrium process results when the

\footnotetext{
${ }^{1}$ email: david.hinde@anu.edu.au

${ }^{2}$ Current address: TIFR, Mumbai, India

${ }^{3}$ Current address: National Superconducting Cyclotron Laboratory, Michigan State University, East Lansing, Michigan 48824, USA

${ }^{4}$ Current address: TIFR, Mumbai, India

${ }^{5}$ Current address: Malmö University, Faculty of Technology and Society, 20506 Malmö, Sweden
} 


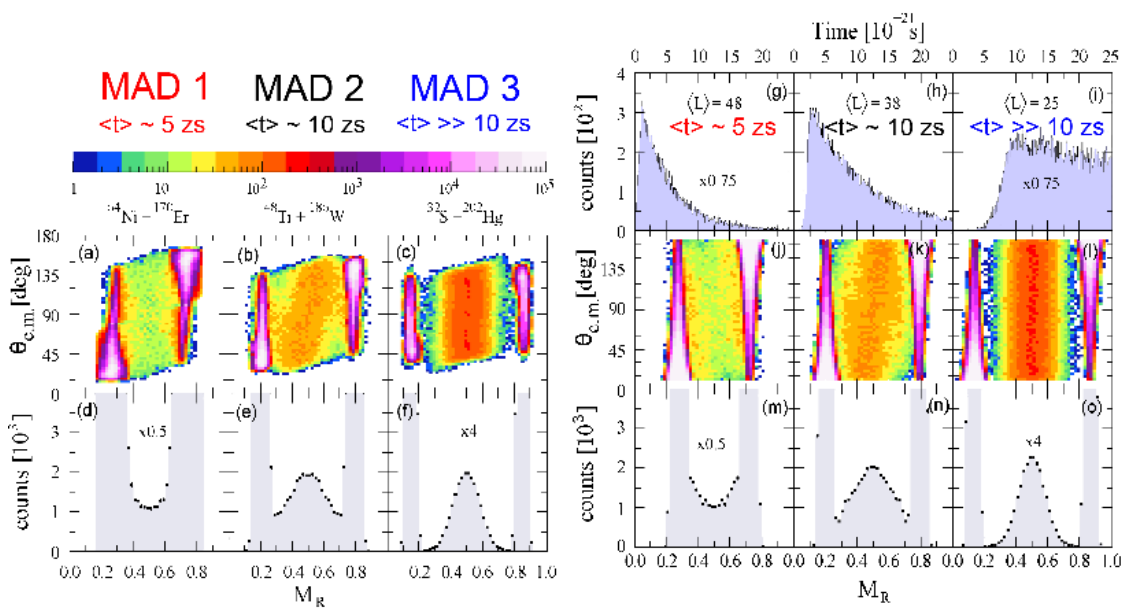

Figure 1: (Color online) Panels (a)-(c) show the experimental MADs, with (d)-(f) showing the projections onto $M_{R}$, for the reactions ${ }^{64} \mathrm{Ni}+{ }^{170} \mathrm{Er},{ }^{48} \mathrm{Ti}+{ }^{186} \mathrm{~W}$ and ${ }^{32} \mathrm{~S}+{ }^{202} \mathrm{Hg}$, which all form ${ }^{234} \mathrm{Cm}$. The multiplicative factors shown scale the $\mathrm{y}$ axis. The simulated MADs for same reactions and beam energies are shown in panels $(\mathrm{g}),(\mathrm{h}),(\mathrm{i})$, with the corresponding $M_{R}$ spectra in panels $(\mathrm{m}),(\mathrm{n}),(\mathrm{o})$, and the sticking time distributions used for the simulations in $(\mathrm{j}),(\mathrm{k}),(\mathrm{l})$. The assigned MAD category for each reaction, and associated approximate mean sticking times are also shown (adapted from Ref. [3]).

combined system formed after capture separates into two (fission-like) fragments in times $\sim 10^{-20} \mathrm{~s}$, before a compact compound nucleus is formed. The probability of quasifission $\left(\mathrm{P}_{Q F}\right)$ can be very large, with the corresponding probability of compound nucleus formation $\left(\mathrm{P}_{C N}=1-\mathrm{P}_{Q F}\right)$ being lower than $10^{-3}$ in unfavourable cases. Understanding the competition between quasifission and fusion is thus very important in predicting the best fusion reactions to use to form new isotopes of heavy and super-heavy elements.

A key quantity characterizing quasifission is its "sticking time" between capture of the two nuclei inside the entrance channel potential barrier [4] and breakup (scission). Quasifission mass-angle distributions (MAD) first measured at GSI in the 1980s [2,5] showed that quasifission timescales could often be shorter than the rotation time of $\sim 10^{-20}$ s. However, subsequently only a few measurements $[6,7]$ were made until recent years, when an extensive series of experiments (using the Australian National University Heavy Ion Accelerator Facility and CUBE spectrometer) were carried out [3,8-16]. The kinematic coincidence technique used in the measurements $[2,3,17]$ provides direct information on the mass-ratio of the fragments at scission; thus, the data are represented in terms of mass ratio $\mathrm{M}_{R}$, rather than pre- or 
post-evaporation masses of the fragments themselves.

Examples of MAD and deduced quasifission sticking time distributions are shown in Fig.1, for reactions forming the compound nucleus ${ }^{234} \mathrm{Cm}[3]$. According to the characteristics of the MAD (minimum mass yield at symmetry, mass-angle correlation with peak yield at symmetry, and no significant mass-angle correlation), they are assigned as type MAD1, MAD2 and MAD3 respectively [3]. There is a clear correlation between the MAD class and the entrance channel charge product. Other entrance channel characteristics are important in determining the sticking times and MAD characteristics, including neutron richness $[14,18]$, and shell structure including static deformation [11] and magic numbers [14].

To improve our quantitative understanding of the role of shell structure in the dynamics of quasifission, we make an analogy with the liquid drop model approach to nuclear masses, in which localized shell effects can be quantified when the underlying smooth (liquid drop) trends are well defined. To define the smooth trends in quasifission, a large number of MAD measurements have been selected, for beam energies somewhat above the capture barrier (typically by $\sim 6 \%$ ). Here the known effects of deformation alignment $[10,11,17,19]$ and shell structure observed in measurements at below-barrier energies $[14,20]$ are much reduced $[11,21,22]$. However the beam energies should not be too far above the capture barriers, otherwise high angular momenta would be introduced in the collisions, which would then not be representative of heavy element formation reactions.

Now we map the reaction outcomes (defined in terms of the MAD classes discussed above) against variables that reflect the balance between nuclear and Coulomb forces during the collision, expected to determine the dynamics of reactions forming very heavy elements, as discussed below.

Ref. [23] proposed that there should be scaling behavior between reactions, on the basis of the schematic "chaotic regime dynamics" model of fusion of heavy nuclei. This is the same model [24] that predicted the "extra push" and "extra-extra-push" kinetic energies needed to overcome the conditional and unconditional saddle-point energies respectively. We have ordered the experimental MAD outcomes as a function of the two scaling parameters expected to most strongly define the reaction outcome: (i) the effective fissility parameter of the entrance channel, $x_{e f f}$, and (ii) the fissility parameter of the compound nucleus, $x_{C N}$. The former is most relevant to necked shapes close to the (generally) mass-asymmetric contact configuration in the entrance channel, whilst the latter applies to shapes without a constricted neck [24], and where the mass-asymmetry degree of freedom is not constrained. 


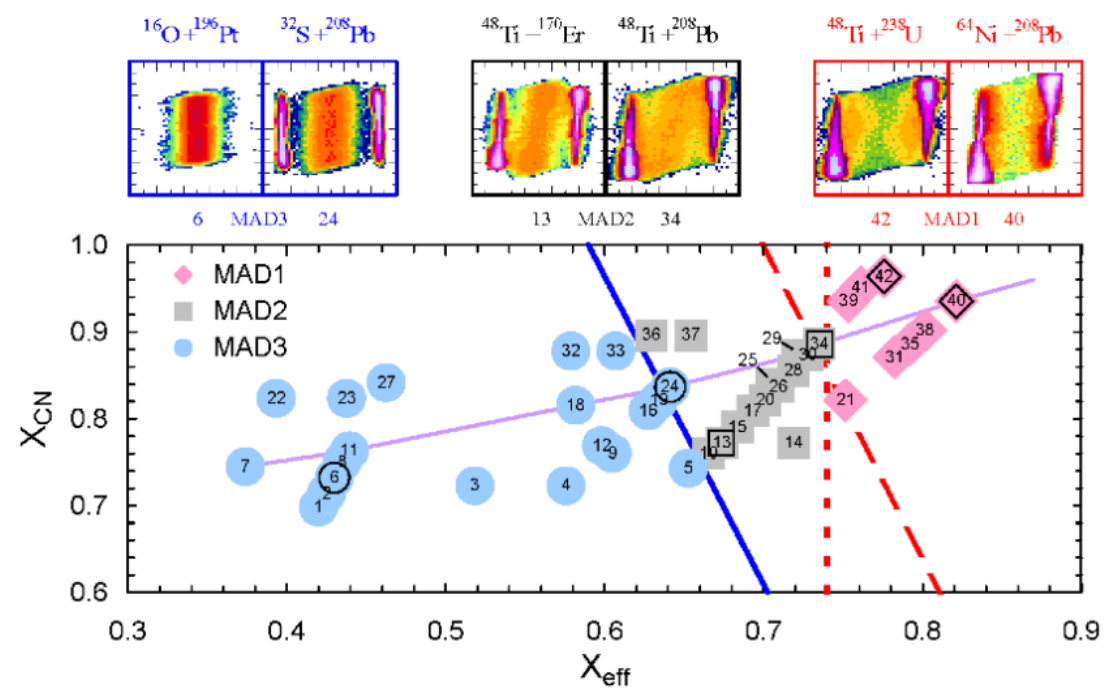

Figure 2: (Color online) The numbers (inside a symbol indicating the classification of MAD observed) refer to the reaction number in Ref. [3], and are plotted as a function of the effective fissility parameter in the entrance channel $x_{e f f}$ and the compound nucleus fissility parameter $x_{C N}$ (see text). The diagonal full line represents the empirical boundary between reactions with no mass-angle correlation (left) and those that have (right). The dashed and dotted lines indicate the uncertainty in the boundary of reactions which no longer exhibit a peak at symmetry in the angle-integrated fission mass distribution. The thin line represents the locus of reactions with ${ }^{208} \mathrm{~Pb}$. Examples of MAD are shown in the panels above, identified by their reaction number.

Fig. 2 shows the experimental MAD class as a function of these two variables. As can be seen, the MAD classes are clustered into groups. The boundary across which a mass-angle correlation becomes significant (between MAD classes 3 and 2) shows a dependence on both variables, with a stronger dependence on the entrance-channel than on the compound nucleus fissility. The full blue line is our estimate of this boundary based on the current data. Mass-angle distributions for reactions on this line should show similar mass-angle correlations, associated with similar reaction trajectories and timescales. The same should be true for reactions on nearby parallel lines. The equation of this boundary line is $0.75 x_{e f f}+0.25 x_{C N}=0.68$, giving $x_{e f f}$ three times the weight of $\mathrm{x}_{C N}$. In terms of the MAD, this defines a mean fissility parameter $\mathrm{x}_{m}$ which seems to give a good characterization of the quasifission observed, at the higher beam and excitation energies chosen here. The thin full line almost orthogonal to the others represents the locus of reactions with the doubly-magic ${ }^{208} \mathrm{~Pb}$. At the above-barrier energies 


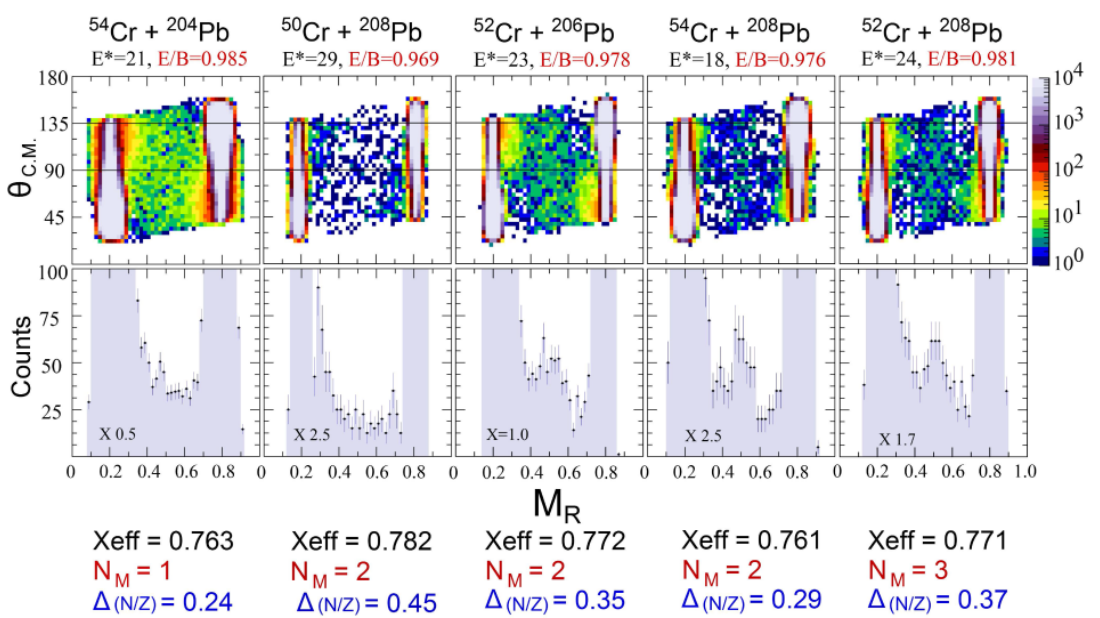

Figure 3: (Color online) MAD and projected mass-ratio distributions for backward angles from $90^{\circ}$ to $135^{\circ}$ (as indicated in the MAD plots), for reactions of ${ }^{50,52,54} \mathrm{Cr}$ isotopes with ${ }^{204,206,208} \mathrm{~Pb}$. Sub-barrier beam energies (denoted by E/B), resulted in the low excitation energies $\mathrm{E}^{*}$. The entrance channel fissility $x_{e f f}$, the number of entrance-channel magic numbers, and the mismatch in $\mathrm{N} / \mathrm{Z}$ values are indicated for each reaction (see text). The reaction outcome changes from a minimum in yield at mass-symmetry (left) to a narrow peak at symmetry, having no evidence of a mass-angle correlation.

chosen, no significant departure from systematic behaviour is seen.

The story is very different at sub-barrier energies. Here it is wellknown that collisions with the tips of deformed nuclei are those that lead to capture [4], and that fission angular distributions [17], mass distributions $[10,13,25]$ and MAD $[11,16]$ point to the changing nature (shorter sticking time) of quasifission under these circumstances. Microscopic TDHF calculations of quasifission masses and angles give a good match [16] to experimental results in reaction ${ }^{40} \mathrm{Ca}+{ }^{238} \mathrm{U}$, across the transition from sub-barrier to above-barrier energies (at which all collision orientations contribute). In sub-barrier reactions with closed-shell nuclei, it has been shown [14] that increasing the "magicity" in the entrance channel results in narrower mass distributions, correlated with a reduced mass-angle correlation, indicative of longer sticking times. Indeed, for the ${ }^{48} \mathrm{Ca}+{ }^{208} \mathrm{~Pb}$ reaction, measured mass distributions [20] appear consistent with a fusion-fission mechanism. These results led to the conclusion that "magicity" plays its strongest role when the $\mathrm{N} / \mathrm{Z}$ values of the projectile and target nuclei are matched. When this is the case, transfer reactions that destroy entrance-channel magicity (as in the 
${ }^{40} \mathrm{Ca}+{ }^{208} \mathrm{~Pb}$ reaction [14]) are minimized, preserving the closed shell nature of the collision partners as long as possible during the merging of the two nuclei [14].

Very recent $\mathrm{ANU}$ results, for the reaction of isotopes of $\mathrm{Cr}$ with $\mathrm{Pb}$, are shown in Fig. 3. These measurements were at sub-barrier energies, resulting in the low excitation energies above the ground-state as indicated. They support the picture from the $\mathrm{Ca}+\mathrm{Pb}$ data. The left three reactions all form the compound nucleus ${ }^{258} \mathrm{Sg}$. The difference between the $\mathrm{N} / \mathrm{Z}$ values of the target and projectile nuclei is denoted by $\Delta_{N / Z}$ in the figure. The panels are ordered from left to right first by the number of magic numbers in the entrance channel $\left(\mathrm{N}_{M}\right)$, and then by $\Delta_{N / Z}$. The left-most reaction has only a single magic number in the entrance channel, and shows a U-shaped mass distribution, consistent with MAD class 1 . With two magic numbers, the reactions with $\Delta_{N / Z}$ closer to zero show a peak at mass-symmetry, associated with an angle-independent ridge in the MAD. With three magic numbers, but less favourable $\Delta_{N / Z}$, similar result is seen. This mass-symmetric peak becomes a smaller and smaller fraction of the total fission yield for $0.3<\mathrm{M}_{R}<0.7$, as the beam energy is increased. These data show a very similar behaviour to the ${ }^{40,44,48} \mathrm{Ca}+{ }^{204,208} \mathrm{~Pb}$ reactions; however, the transition from a U-shaped mass distribution to a narrow mass-symmetric mass distribution is in some ways an even more drastic change in reaction outcome. The very sudden change in outcome, depending on neutron number and magicity, will be a severe challenge for models of quasifission to reproduce. And yet it is this level of sensitivity that models must strive to reproduce, to optimise experimental opportunities to create new isotopes of superheavy elements.

\section{Acknowledgments}

The authors acknowledge operations support for the ANU Heavy Ion Accelerator Facility from NCRIS, and support from Dr. N. Lobanov and Dr. T. Kibedi and the ANU Heavy Ion Accelerator Facility staff in operating the Linac. Financial support from ARC grants DP130101569, DP140101337, FL110100098, FT120100760 and DE140100784 is acknowledged.

\section{References}

[1] D.J. Hinde, M. Dasgupta, A. Mukherjee, Phys. Rev. Lett. 89, 282701 (2002) 
[2] J. Tōke, B. Bock, G.X. Dai, A. Gobbi, S. Gralla, K.D. Hildenbrand, J. Kuzminski, W. Müller, A. Olmi, H. Stelzer, Nucl. Phys. A 440, 327 (1985)

[3] R. du Rietz, E. Williams, D.J. Hinde, M. Dasgupta, M. Evers, C.J. Lin, D.H. Luong, C. Simenel, A. Wakhle, Phys. Rev. C 88, 054618 (2013)

[4] M. Dasgupta, D.J. Hinde, N. Rowley, A.M. Stefanini, Annu. Rev. Nucl. Part. Sci. 48, 401 (1998)

[5] W.Q. Shen, J. Albinski, A. Gobbi, S. Gralla, K.D. Hildenbrand, N. Herrmann, J. Kuzminski, W.F.J. Müller, H. Stelzer, J. Tōke et al., Phys. Rev. C 36, 115 (1987)

[6] J. Velkovska, C.R. Morton, R.L. McGrath, P. Chung, I. Diószegi, Phys. Rev. C 59, 1506 (1999)

[7] B.B. Back, P.B. Fernandez, B.G. Glagola, D. Henderson, S. Kaufman, J.G. Keller, S.J. Sanders, F. Videbæk, T.F. Wang, B.D. Wilkins, Phys. Rev. C 53, 1734 (1996)

[8] R.G. Thomas, D.J. Hinde, D. Duniec, F. Zenke, M. Dasgupta, M.L. Brown, M. Evers, L.R. Gasques, M.D. Rodriguez, A. Diaz-Torres, Phys. Rev. C 77, 034610 (2008)

[9] R. Rafiei, R.G. Thomas, D.J. Hinde, M. Dasgupta, C.R. Morton, L.R. Gasques, M.L. Brown, M.D. Rodriguez, Phys. Rev. C 77, 024606 (2008)

[10] D.J. Hinde, R. du Rietz, M. Dasgupta, R.G. Thomas, L.R. Gasques, Phys. Rev. Lett. 101, 092701 (2008)

[11] D.J. Hinde, R.G. Thomas, R. du Rietz, A. Diaz-Torres, M. Dasgupta, M.L. Brown, M. Evers, L.R. Gasques, R. Rafiei, M.D. Rodriguez, Phys. Rev. Lett. 100, 202701 (2008)

[12] R. du Rietz, D.J. Hinde, M. Dasgupta, R.G. Thomas, L.R. Gasques, M. Evers, N. Lobanov, A. Wakhle, Phys. Rev. Lett. 106, 052701 (2011)

[13] C.J. Lin, R. du Rietz, D.J. Hinde, M. Dasgupta, R.G. Thomas, M.L. Brown, M. Evers, L.R. Gasques, M.D. Rodriguez, Phys. Rev. C 85, 014611 (2012)

[14] C. Simenel, D.J. Hinde, R. du Rietz, M. Dasgupta, M. Evers, C.J. Lin, D.H. Luong, A. Wakhle, Phys. Lett. B 710, 607 (2012) 
[15] E. Williams, D.J. Hinde, M. Dasgupta, R. du Rietz, I.P. Carter, M. Evers, D.H. Luong, S.D. McNeil, D.C. Rafferty, K. Ramachandran et al., Phys. Rev. C 88, 034611 (2013)

[16] A. Wakhle, C. Simenel, D.J. Hinde, M. Dasgupta, M. Evers, D.H. Luong, R. du Rietz, E. Williams, Phys. Rev. Lett. 113, 182502 (2014)

[17] D.J. Hinde, M. Dasgupta, J.R. Leigh, J.C. Mein, C.R. Morton, J.O. Newton, H. Timmers, Phys. Rev. C 53, 1290 (1996)

[18] K. Hammerton, Z. Kohley, D.J. Hinde, M. Dasgupta, A. Wakhle, E. Williams, V.E. Oberacker, A.S. Umar, I.P. Carter, K.J. Cook et al., Phys. Rev. C 91, 041602 (2015)

[19] D.J. Hinde, M. Dasgupta, J.R. Leigh, J.P. Lestone, J.C. Mein, C.R. Morton, J.O. Newton, H. Timmers, Phys. Rev. Lett. 74, 1295 (1995)

[20] E.V. Prokhorova, A.A. Bogachev, M.G. Itkis, I.M. Itkis, G. Knyazheva, N.A. Kondratiev, E.M. Kozulin, L. Krupa, Y.T. Oganessian, I.V. Pokrovsky et al., Nucl. Phys. A 802, 45 (2008)

[21] K. Nishio, H. Ikezoe, S. Mitsuoka, I. Nishinaka, Y. Nagame, Y. Watanabe, T. Ohtsuki, K. Hirose, S. Hofmann, Phys. Rev. C 77, 064607 (2008)

[22] I.M. Itkis, E.M. Kozulin, M.G. Itkis, G.N. Knyazheva, A.A. Bogachev, E.V. Chernysheva, L. Krupa, Y.T. Oganessian, V.I. Zagrebaev, A.Y. Rusanov et al., Phys. Rev. C 83, 064613 (2011)

[23] J. Blocki, H. Feldmeier, W. Swiatecki, Nucl. Phys. A 459, 145 (1996)

[24] S. Bjornholm, W. Swiatecki, Nucl. Phys. A 391(2), 471 (1982)

[25] K. Nishio, H. Ikezoe, I. Nishinaka, S. Mitsuoka, K. Hirose, T. Ohtsuki, Y. Watanabe, Y. Aritomo, S. Hofmann, Phys. Rev. C 82, 044604 (2010) 